\title{
SEMISIMPLE CLASSES OF ALTERNATIVE RINGS
}

\author{
by T. ANDERSON and R. WIEGANDT*
}

(Received 21st June 1979)

\section{Introduction}

Recently A. D. Sands [7] solved a problem posed in [6], and characterised the semisimple classes of associative rings as classes being regular, coinductive and closed under extensions. It is the purpose of this note to prove the same assertion for alternative rings. This result is perhaps not surprising, nevertheless its proof cannot be considered an easy one, and it requires a technique of dealing with ideals of ideals. In addition, semisimple classes of hereditary radicals and those of supernilpotent radicals will be characterised as easy consequences of our theorem.

\section{Preliminaries}

A not necessarily associative ring $A$ is called an alternative ring if

$$
(x x) y=x(x y) \text { and } y(x x)=(y x) x
$$

hold for every $x, y \in A$. The associator $(x, y, z)$ is defined as

$$
(x, y, z)=(x y) z-x(y z)
$$

and for alternative rings we have the equalties

$$
(x, y, z)=(y, z, x)=(z, x, y)=-(x, z, y)=-(z, y, x)=-(y, x, z) .
$$

We shall make use of the following assertions.

Proposition 1. (cf. [1]: (7)). In an alternative ring $A$ for any elements $a, b, c, u \in A$ the equality

$$
b(c, a, u)+c(b, a, u)=(c, a b, u)+(b, a c, u)
$$

Propositions 2. (cf. [1]: (9')). If $C \triangleleft B \triangleleft A$ and $a \in A$, then $a C+C \triangleleft B$.

Proposition 3. If $C \triangleleft B \triangleleft A, a \in A$ and $x \in C$, then $[(a x)(a C)+C] \triangleleft B$.

For the proof we refer to [5], p. 177.

* This research was carried out during a stay of the second author at the University of British Columbia. Both authors gratefully acknowledge the support of the National Research Council of Canada. 
Proposition 4. If $C \triangleleft B \triangleleft A, a \in A$ and $x \in C$, then $[(a x)(a C)+C]^{2} \subseteq C$.

The proof can be found in [5], p. 176.

Proposition 5. If $C \triangleleft B \triangleleft A, a \in A$ and $x \in C$ then the mapping

$$
\phi: C \rightarrow[(a x)(a C)+C] / C
$$

defined by $\phi(z)=(a x)(a z)+C$, is an epimorphism.

The proof of the assertion is given in [5], p. 176 .

Proposition 6. If for every $x \in C$ the previously defined mapping $\phi$ maps $C$ onto 0 , then the mapping

$$
\psi: C \rightarrow(a C+C) / C
$$

defined by $\psi(z)=a z+C$, is an epimorphism. Moreover $(a C+C) / C$ is a zero-ring.

For the proof we refer to [5], p. 176.

Next, suppose that $C \triangleleft B \triangleleft A, a \in A, x \in C$ and define the set $F$ as

$$
F=\{u \in C \mid(a x)(a u) \in C\} \text {. }
$$

Proposition 7. If $u \in F$, and $b \in B$, then $(a x)(a, b, u) \in C$.

Proof. Using Proposition 1 we get

$$
(a x)(a, b, u)=-(a x)(b, a, u)=b(a x, a, u)-(b, a(a x), u)-(a x, a b, u) .
$$

However,

$$
b(a x, a, u)=b[((a x) a) u]-b[(a x)(a u)] \in B(B C)+B C \subseteq C
$$

and the other terms $(b, a(a x), u)$ and $(a x, a b, u)$ are in $(B, B, C) \subseteq C$. Thus the assertion is proved.

Proposition 8. $F \triangleleft B$.

Proof. $F$ is clearly an additive subgroup of $B$. Let $u \in F$ and $b \in B$ be arbitrary elements. Then applying Proposition 7 we get

$$
(a x)[a(b u)]=-(a x)(a, b, u)+(a x)[(a b) u)] \in C+B(B C) \subseteq C .
$$

Thus $b u \in F$. Further,

$$
(a x)[a(u b)]=(a x)[(a u) b]-(a x)(a, u, b)
$$

and by Proposition 7 the second term is in $C$. Checking the first term by applying Proposition 1, we have

$$
\begin{aligned}
(a x)[(a u) b] & =[(a x)(a u)] b-(a x, a u, b) \\
& =[(a x)(a u)] b+(u, a(a x), b)-(a x)(u, a, b)-u(a x, a, b) .
\end{aligned}
$$


Hence by Proposition 7 it follows that

$$
(a x)[(a u) b] \in C B+(C, B, B)+C+C(B, A, B) \subseteq C .
$$

Thus $(a x)[a(u b)] \in C$. Hence $u b \in F$, which completes the proof that $F$ is an ideal in B.

\section{Semisimple classes}

A class $\boldsymbol{C}$ of (not necessarily associative) rings is called a universal class if $\boldsymbol{C}$ is closed under homomorphisms and is hereditary, that is, $B \triangleleft A \in \boldsymbol{C}$ implies $B \in \boldsymbol{C}$. All classes considered in this paper are supposed to be isomorphically closed.

As is well known, a class $\boldsymbol{S}$ in a universal class of rings is a semisimple class, if $\boldsymbol{S}$ satisfies the following two conditions:

(A) $S$ is a regular class, that is, if $A \in S$ then every non-zero ideal of $A$ has a non-zero homomorphic image in $\boldsymbol{S}$;

(B) If every non-zero ideal of a ring $A$ has a non-zero homomorphic image in $S$, then $A \in S$.

Our goal is to prove the following characterization of semisimple classes in a universal class of alternative rings.

Theorem. A subclass $\mathbf{S}$ of alternative rings is a semisimple class if and only if $\mathbf{S}$ satisfies the following three conditions:

(i) $\boldsymbol{S}$ is a regular class,

(ii) $\boldsymbol{S}$ is closed under extensions, that is $B \in \boldsymbol{S}$ and $A / B \in \boldsymbol{S}$ imply $A \in \boldsymbol{S}$,

(iii) $S$ is coinductive, that is whenever a ring $A$ contains a descending chain of ideals $B$, such that $\cap B_{i}=0$ and $A / B_{i} \in S$ for each $i$, then $A \in S$.

Proof. It is known that conditions (i), (ii) and (iii) are necessary (cf. for instance [3], Theorems 12 and 13).

Let us suppose that a class $S$ satisfies (i), (ii) and (iii). The theorem will be proved if we exhibit the validity of condition (B). However, we first note that the class $\boldsymbol{S}$ satisfies the following closure property with respect to ideals.

$$
0 \neq I \triangleleft A \in S \text { and } A^{2}=0 \Rightarrow I \in S .
$$

Indeed, in view of (i), $I$ has a non-zero homomorphic image in $S$ and by (iii) and Zorn's Lemma, we may assume $J$ is an ideal of $I$ which is minimal with respect to the property $I / J \in S$. If $0 \neq J$ then $J \triangleleft A$ (because $A^{2}=0$ ), hence by (i) $J$ has an ideal $K$ such that $0 \neq J / K$ and $J / K \in S$. Clearly, $K$ is an ideal of $I$ and as $I / J \cong I / K / J / K, I / K \in S$ because of (ii). However, this contradicts the minimality of $J$. Therefore $J=0$ and $I \in S$, which proves (1).

We may now improve the result (1) to the following:

$$
0 \neq I \triangleleft A \in S \text { and } I^{2}=0 \Rightarrow I \in S \text {. }
$$

To see this, choose $J$ as before. If $J \triangleleft A$ then the preceding argument shows $I \in S$. Therefore we assume $a J \nsubseteq J$ for some $a \in A$ (the case $J a \nsubseteq J$ can be handled by the 
same sort of argument). Because of Proposition 2, we have $0 \neq \frac{J+a J}{J} \triangleleft I / J$. As $I^{2}=0$ and $I / J \in S, \frac{J+a J}{J} \in S$ on account of (1). Now consider the map $\psi: J \rightarrow \frac{J+a J}{J}$ defined by $\psi(j)=J+a j, j \in J$. Since $I^{2}=0, \psi$ is an epimorphism and ker $\psi$ is an ideal of $I$. Then as $I / J=\frac{I / \operatorname{ker} \psi}{J / \operatorname{ker} \psi}, I / \operatorname{ker} \psi \in S$ because of (ii). This contradicts the minimality of $J$ since $J \neq \operatorname{ker} \psi$. Therefore $0=J$ and $I \in \mathbf{S}$.

Now we shall verify that $S$ satisfies the condition (B). For this end let $A$ be a ring such that every non-zero ideal of $A$ has a non-zero homomorphic image in $S$, but $A$ is not contained in $S$. This hypothesis on $A$ will lead to a contradiction.

Because of condition (i), $\boldsymbol{A}$ has a non-zero homomorphic image in $\boldsymbol{S}$ and by (iii) and Zorn's Lemma we may assume that $B$ is an ideal of $A$ minimal with respect to the property $A / B \in S$. By hypothesis $B \neq 0$; hence (i) implies that $B$ has a non-zero homomorphic image $B / C$ in $S$ and again in view of (iii) and Zorn's Lemma, we may suppose that $C$ is minimal with respect to the property $B / C \in S$.

We are going to prove that $C \triangleleft A$. Assume that this is not the case. Then there exists an element $a \in A$ such that $a C \nsubseteq C$ or $C a \nsubseteq C$. We shall deal only with the case $a C \nsubseteq C$, for the other case can be handled analogously.

For any fixed $x \in C$ we note that

$$
[C+(a x)(a C) / C] \triangleleft B / C
$$

and

$$
[C+(a x)(a C) / C]^{2}=0
$$

because of Propositions 3 and 4 . Hence it follows from our previous observation (2) that $[C+(a x)(a C) / C] \in S$. In view of Proposition 5, the map

$$
\phi_{x}: C \rightarrow[C+(a x)(a C) / C]
$$

defined $\phi_{x}(z)=C+(a x)(a z)$ is an epimorphism and $C / \operatorname{ker} \phi_{x} \in \mathbf{S}$. However, ker $\phi_{x}$ is an ideal of $B$ because of Proposition 8 . As $B / C \cong \frac{B / \operatorname{ker} \phi_{x}}{C / \operatorname{ker} \phi_{x}}$ it follows from (ii) that $B /$ ker $\phi_{x} \in \boldsymbol{S}$. However since $C$ was minimal with respect to the property $B / C \in \boldsymbol{S}$, this implies $C=\operatorname{ker} \phi_{x}$, that is, $\phi_{x}=0$.

In proceeding to show that $C \triangleleft A$ we consider the mapping

$$
\psi: C \rightarrow C+a C \neq 0
$$

defined by $\psi(z)=C+a z$. Proposition 6 is applicable, showing that $\psi$ maps $C$ homomorphically onto $C+a C / C$ and that $C+a C / C$ is a zero-ring. Since by Proposition $2 C+a C / C$ is an ideal of $B / C$, it follows from (2) that $C+a C / C \in \boldsymbol{S}$; hence $C /$ ker $\psi \in \mathbf{S}$.

We claim now that ker $\psi \triangleleft B$. Noting that ker $\psi=\{t \in C \mid a t \in C\}$, take $t \in \operatorname{ker} \psi$ and $b \in B$. Then $a(t b)=(a t) b-(a, t, b)=(a t) b-(b, a, t)=(a t) b-(b a) t+b(a t) \in C B+B C+$ $B C \subseteq C$. This means $t b \in \operatorname{ker} \psi$ and $(a, t, b) \in C$. Thus $a(b t)=(a b) t-(a, b, t)=$ $(a b) t+(a, t, b) \in B C+C \subseteq C$ holds, showing $b t \in \operatorname{ker} \psi$. Hence ker $\psi \triangleleft B$ is established. 
Altogether we have $0 \neq C / \operatorname{ker} \psi \in S$ as well as $B / C \in S$. Since $B / C \cong \frac{B / \operatorname{ker} \psi}{C / \operatorname{ker} \psi}$ we conclude from (ii) that $B /$ ker $\psi \in S$ which contradicts the choice of $C$. Therefore $a C \subseteq C$ for every $a \in A$.

By analogous considerations one gets $C A \subseteq A$. Hence $C$ is an ideal of $A$.

Now we have

$$
\frac{A / C}{B / C} \cong A / B \in S
$$

and $B / C \in S$, so condition (ii) implies $A / C \in S$, contradicting the minimality of $B$. This contradiction proves that the hypothesis $A \notin S$ is impossible. Thus $\boldsymbol{S}$ satisfies also condition $(B)$ and so $S$ is a semisimple class.

Remark 1. The scheme of our proof was somewhat simpler than that of Sands' proof, inasmuch as we showed directly that the class considered satisfied condition $(B)$. Doing so, we could save the investigation of further mappings.

Corollary 1. A class $\boldsymbol{S}$ of alternative rings is a semisimple class if and only if $\boldsymbol{S}$ is regular, closed under extensions and subdirect sums.

The next assertion sharpens van Leeuwen's Theorem. Let us recall that an ideal $L$ of a ring $A$ is large, if $L \cap I \neq 0$ for every ideal $I \neq 0$ of $A$.

Corollary 2. A class $\mathbf{S}$ of alternative rings is the semisimple class of a hereditary radical if and only if $\boldsymbol{S}$ is regular, closed under subdirect sums and satisfies condition

$$
\text { If } L \text { is a large ideal of } A \text { and } L \in S \text {, then also } A \in S
$$

Proof. In view of [4], any class that is subdirectly closed and satisfies $(\lambda)$ is closed under extensions. Hence by the Theorem it is a semisimple class. Then by [1] the class is also hereditary, and so van Leeuwen's Theorem (see [6]) is applicable.

We also give a new characterisation of semisimple classes of supernilpotent radicals. For an alternative ring $A$ let us define

$$
A^{(0)}=A \quad \text { and } \quad A^{(n)}=\left(A^{(n-1)}\right)\left(A^{(n-1)}\right)
$$

for $n=1,2, \ldots$ A ring $A$ is solvable, if $A^{(n)}=0$ for some $n \geq 1$. A radical class is said to be hypersolvable, if it contains all solvable rings. Hereditary and hypersolvable radicals are referred to as supersolvable radicals. A class $C$ of rings is said to be weakly homomorphically closed, if $B \triangleleft A \in C$ and $B^{2}=0$ imply $A / B \in C$.

Corollary 3. A proper subclass $\boldsymbol{S}$ of the variety of all alternative or associative rings is a semisimple class of a supersolvable radical if and only if $\mathbf{S}$ is regular, subdirectly closed, weakly homomorphically closed, and satisfies condition $(\lambda)$. 4.

Proof. The assertion is an immediate consequence of Corollary 2 and [2], Corollary 
Remark. Neither in Corollary 2 nor in Corollary 3 can the condition that $\boldsymbol{S}$ is subdirectly closed be replaced by demanding that $\boldsymbol{S}$ is coinductive. Let us consider the class $\boldsymbol{C}$ consisting of 0 and a single simple ring with unity. $\boldsymbol{C}$ is obviously a regular class which is coinductive, weakly homomorphically closed, and satisfies condition $(\lambda)$, though $\boldsymbol{C}$ fails to be subdirectly closed. On the other hand the upper radical of $\boldsymbol{C}$ is, of course, a supersolvable radical.

\section{REFERENCES}

1. T. Anderson, N. Divinsky and A. Suléinski, Hereditary radicals in associative and alternative rings, Canad. J. Math. 17 (1965), 594-603.

2. T. Anderson and R. Wiegandt, Weakly homomorphically closed semi-simple classes, Acta Math. Acad. Sci. Hungar. 34 (1979), 329-336.

3. W. G. LeAvitT, The general theory of radicals (Univ. of Nebraska, Lincoln, 1970).

4. L. C. A. van Leeuwen, Properties of semisimple classes, J. Nat. Sci. \& Math., Lahore 15 (1975), 59-67.

5. L. C. A. van Leeuwen, C. Roos and R. Wiegandt, Characterizations of semisimple classes, J. Austral. Math. Soc. (Series A) 23 (1977), 172-182.

6. L. C. A. VAN LEEUWEN and R. WIEGANDT, Radicals, semisimple classes and torsion theories, Acta Math. Acad. Sci. Hungar. 36 (1980), 37-47.

7. A. D. SAnds, A characterization of semisimple classes, Math. Soc. (2) 24 (1981), 5-7.

Department of Mathematics

University of British Columbia

VANCOUVER, B.C.

CANADA

Mathematical InSTITUTE

Hungarian ACADEMY OF SCIENCE

ReALTANODA u. 13-15

H-1053 BuDAPEST. 